\title{
ENERGY DISTRIBUTION OF A CHARGED BLACK HOLE WITH A MINIMALLY COUPLED SCALAR FIELD
}

\author{
PAUL HALPERN \\ Department of Mathematics, Physics and Statistics, \\ University of the Sciences in Philadelphia, 600 S. 43rd St., \\ Philadelphia, PA. 19104, USA \\ p.halper@usip.edu
}

\begin{abstract}
Using three different energy-momentum complexes, the Einstein, Landau-Lifshitz, and Papapetrou prescriptions, we calculate the energy of an electrically charged black hole exact solution with a self-interacting, minimally-coupled scalar field and the asymptotic region locally an anti-deSitter spacetime. Writing the metric in Kerr-Schild Cartesian coordinates, we demonstrate that this metric belongs to the Kerr-Schild class of solutions. Applying each of the three energy-momentum prescriptions and comparing the results, we find consistency among these complexes, suggesting their utility as localized measures of energy.

Keywords: black hole; AdS spacetime, Reissner-Nordström solution; no-hair theorem; energy-momentum complex.
\end{abstract}

PACS numbers: 04.20.Cv, 04.40.Nr, 04.70.Bw

\section{Introduction}

One of Einstein's challenges in formulating the general theory of relativity was incorporating the energy and momenta of gravitational fields along with those of matter and non-gravitational fields into a local, well-defined conserved quantity [1]. Einstein's efforts along these lines led him to propose an energymomentum complex that would include all fields and obey a conservation law [2]. Non-tensorial in nature, the Einstein complex is highly dependent on the coordinate system used. In the case of a flat, empty space-time, for example, the Einstein complex vanishes for quasi-Cartesian coordinates but is infinite for spherical coordinates.

The Einstein energy-momentum complex is asymmetric in its indices, precluding the definition of a conserved angular momentum and leading Landau and Lifshitz [3], Papapetrou [4], Weinberg [5] and others to construct alternative formulations. In general, these favor quasi-Cartesian coordinate systems. Yet another prescription, developed by Møller [6], is uniquely designed to be independent of the choice of coordinate system.

The multiplicity of energy-momentum complexes and their particular constraints once deterred theorists from their use. However, in recent years interest has been revived due to a number of promising findings about consistent ways 
of defining energy localization. In 1990 Bondi argued that energy could at least in principle be localized [7]. The same year Virbhadra found that different energy-momentum complexes when applied to the same metric furnish reasonable, consistent results [8].

These results were extended in 1996 when Aguirregabiria, Chamorro and Virbhadra established that all metrics of the Kerr-Schild class (the Schwarzchild, Reissner-Nordström and many other well-known solutions) have unique, welldefined energy and momentum distributions even when different complexes are applied. It remains an open question why certain classes of metrics yield identical energy-momentum distributions for a range of complexes, while others do not produce such consistency. Nevertheless, as Aguirregabiria, Chamorro and Virbhadra wrote, "different pseudotensors giving the same results for local quantities... does not seem to be accidental," [9] suggesting that certain symmetries present in particular metrics lend themselves well to consistent definitions of local conservation laws. Resolving this issue has been the subject of continuing investigation, including cosmological as well as stationary solutions.

In 1994 Rosen applied the Einstein energy-momentum complex to a closed Friedmann-Robertson-Walker (FRW) cosmology, found a zero value for its energy integrated over the full range of spatial coordinates, and speculated that the total energy of the universe is identically zero [10]. Other researchers have extended these results to other complexes [11], anisotropic cosmologies [12-17], and teleparallel gravity (a modification of general relativity) [18-21].

Black holes and related objects have been a much studied application of energy-momentum complexes. Using various complexes, Vagenas has examined the dyadosphere of Reissner-Nordström black holes [22], investigated stringy black holes [23], considered the Baados, Teitelboim, and Zanelli (BTZ) metric (a rotating $2+1$ dimensional black hole solution) [24], and evaluated the energy of radiating charged particles [25]. Xulu has also calculated the energy of stringy black holes [26]. Radinschi and her colleagues [27, 28], as well as Gad [29], have also investigated a variety of cases for $2+1$ dimensional and stringy black holes. Chamorro and Virbhardra [30], Vagenas [31] and Xulu [32] have each calculated the energy distribution of charged black holes with a dilaton field. Yet other researchers have looked at black plane solutions [33] and a stationary beam of light $[34,35]$

Much of this work has been stimulated by a 1999 paper by Virbhadra suggesting that the energy-momentum complexes of Einstein, Landau and Lifshitz, Papapetrou and Weinberg yield the same results for metrics more general than the Kerr-Schild class if they are expressed in terms of Kerr-Schild Cartesian coordinates [36]. Virbhadra emphasized that although the possibility of a local definition of energy and momentum is subject to debate and these complexes 
are not covariant, nevertheless they offer promise because of such consistency and also due to the fact that they each satisfy local conservation laws in all coordinate systems. More recently, Xulu has extended this work by applying the Bergmann-Thomson energy-momentum complex to solutions more general than the Kerr-Schild class [37].

Along these lines it is interesting to apply several different energy-momentum complexes to a newly found solution describing a charged black hole with scalar "hair."

\section{Charged black holes with scalar hair}

In the early 1970s, Wheeler and others, in considering stable black hole solutions of the Einstein-Maxwell equations, formulated the well-known "nohair theorem" (from Wheeler's maxim: "Black holes have no hair") limiting the observable properties of a black hole to three parameters: mass, electric charge and angular momentum [38-40]. The black hole's event horizon cloaks any additional information that could betray its origin.

In recent years, however, certain exceptions to the no hair theorem have been revealed. Numerical solutions that circumvent its restrictions were found by Torii, Maeda and Narita, Winstanley and others [41-44]. Exact hairy solutions with a scalar field were discovered by Martínez, Troncoso and Zanelli [45] and later by Zloshchastiev [46]. Koutsoumbas, Musiri, Papantonopoulos and Siopsis explored the perturbative behavior of black holes with scalar hair and identified a second-order phase transition [47]. Radu and Winstanley argued for the existence of solutions with a conformally coupled scalar field in four or more dimensions if a negative cosmological constant is present [48].

In 2006, Martínez and Troncoso identified a new exact solution describing a black hole in $3+1$ dimensions with electric charge and a self-interacting scalar field minimally coupled to electromagnetism and gravitation [49]. The scalar field generates an effective negative cosmological constant, rendering the black hole's event horizon a surface of constant negative curvature, and making its asymptotic behavior locally that of an anti-deSitter (AdS) spacetime. An anti-deSitter spacetime is a maximally symmetric solution with a negative cosmological constant and possessing constant negative curvature. A number of researchers have studied the thermodynamic properties of black holes with asymptotic AdS regions [50-54]. In line with these earlier findings, Martínez and Troncoso have established that their solution has a well-defined fixed temperature, independent of horizon size, and an entropy that obeys the usual area law. Their solution is massless, regardless of the value of the charge. Although the influence of the scalar field represents a circumvention of the no-hair theo- 
rem, Martínez and Troncoso found that their solution has a finite probability of decaying into a black hole without a scalar field and hence without hair.

Martínez and Troncoso's solution obeys the standard Einstein-Maxwell field equations modified by the addition of a minimally coupled, self-interacting scalar field, namely:

$$
\begin{aligned}
G_{\mu \nu} & =8 \pi G\left(T_{\mu \nu}{ }^{(E M)}+T_{\mu \nu}{ }^{(\phi)}\right) \\
\partial_{\nu}\left(\sqrt{-g} F^{\mu \nu}\right) & =0 \\
\square \phi & =\frac{d V}{d \phi}
\end{aligned}
$$

where the electromagnetic and scalar parts of the stress-energy tensor are:

$$
\begin{aligned}
T_{\mu \nu}{ }^{(E M)} & =-\frac{1}{4 \pi}\left(F_{\alpha}^{\mu} F^{\alpha \mu}+\frac{1}{4} g^{\mu \nu} F^{\alpha \beta} F_{\alpha \beta}\right) \\
T_{\mu \nu}{ }^{(\phi)} & =\partial_{\mu} \phi \partial_{\nu} \phi-\frac{1}{2} g_{\mu \nu} g^{\alpha \beta} \partial_{\alpha} \phi \partial_{\beta} \phi-g_{\mu \nu} V(\phi)
\end{aligned}
$$

and the potential for the self-interacting scalar field has the form:

$$
V(\phi)=-\frac{3}{8 \pi G l^{2}} \cosh ^{4}\left(\sqrt{\frac{4 \pi G}{3}} \phi\right)
$$

For $\phi=0$ this potential reaches a global maximum. The non-zero expectation value yields an effective negative cosmological constant that can be expressed in terms of the AdS radius as:

$$
\Lambda=-\frac{3}{l^{2}}
$$

The field equations are satisfied by the metric:

$$
\begin{aligned}
d s^{2} & =\left(1+\frac{G q^{2}}{r^{2}}\right)^{-1}\left(\frac{r^{2}}{l^{2}}-1+\frac{G q^{2}}{l^{2}}\right) d t^{2} \\
& -\left(1+\frac{G q^{2}}{r^{2}}\right) \quad\left(\frac{r^{2}}{l^{2}}-1+\frac{G q^{2}}{l^{2}}\right)^{-1} d r^{2}-r^{2} d \sigma^{2}
\end{aligned}
$$

with $q$ representing the electric charge and the electromagnetic potential and scalar field set to be:

$$
\begin{aligned}
A & =-\frac{q}{\sqrt{r^{2}+G q^{2}}} d t \\
\phi & =\sqrt{\frac{3}{4 \pi G}} \operatorname{arctanh} \sqrt{\frac{G q^{2}}{r^{2}+G q^{2}}}
\end{aligned}
$$




\section{Evaluating the energy using Einstein's energy-momentum complex}

It is interesting to use Einstein's prescription to evaluate the energy distribution of Martínez and Troncoso's metric. Along the lines of Virbhadra's findings in [40], we express the metric in Kerr-Schild Cartesian coordinates, choosing natural units such that $G=1$. We define a new time coordinate $T$ such that:

$$
d T=\left(1+\frac{q^{2}}{r^{2}}\right)^{-\frac{3}{2}} d t+\left[\left(1+\frac{q^{2}}{r^{2}}\right)^{-2}\left(\frac{r^{2}}{l^{2}}-1+\frac{q^{2}}{l^{2}}\right)^{-1}-1\right] d r
$$

The metric (8) can now be written in Kerr-Schild-Cartesian form as:

$$
d s^{2}=d T^{2}+\left[\left(1+\frac{q^{2}}{r^{2}}\right)^{2}\left(\frac{r^{2}}{l^{2}}-1+\frac{q^{2}}{l^{2}}\right)-1\right][d T+d r]^{2}-d x^{2}-d y^{2}-d z^{2}
$$

with the three Cartesian spatial coordinates expressed in terms of the spherical coordinates in the usual way:

$$
\begin{aligned}
& x=r \sin \theta \cos \phi \\
& y=r \sin \theta \sin \phi \\
& z=r \cos \theta
\end{aligned}
$$

Einstein's prescription defines the local energy-momentum density as:

$$
\theta_{i}{ }^{k}=\frac{1}{16 \pi} H_{i}^{k l}, l
$$

where the superpotentials $H_{i}{ }^{k l}$ are given by:

$$
H_{i}^{k l}=\frac{g_{i n}}{\sqrt{-g}}\left[-g\left(g^{k n} g^{l m}-g^{l n} g^{k m}\right)\right]_{, m}
$$

This complex has the antisymmetric property that:

$$
H_{i}{ }^{k l}=-H_{i}^{l k}
$$

We substitute the metric components (12) into (17) and find that the relevant values of the superpotentials are:

$$
\begin{aligned}
& H_{0}{ }^{01}=\frac{q^{4}+2 q^{2} r^{2}+2 r^{4}}{r^{6}}-\frac{2 x\left(q^{2}+r^{2}\right)^{3}}{l^{2} r^{6}} \\
& H_{0}{ }^{02}=\frac{q^{4}+2 q^{2} r^{2}+2 r^{4}}{r^{6}}-\frac{2 y\left(q^{2}+r^{2}\right)^{3}}{l^{2} r^{6}} \\
& H_{0}{ }^{03}=\frac{q^{4}+2 q^{2} r^{2}+2 r^{4}}{r^{6}}-\frac{2 z\left(q^{2}+r^{2}\right)^{3}}{l^{2} r^{6}}
\end{aligned}
$$


The energy-momentum components can be found by integrating the energymomentum density over the volume under consideration:

$$
P_{i}=\iiint \theta_{i}{ }^{0} d x^{1} d x^{2} d x^{3}
$$

Through Gauss's theorem we can express this as a surface integral:

$$
P_{i}=\frac{1}{16 \pi} \iint H_{i}^{0 \alpha} \mu_{\alpha} d S
$$

where $\mu_{\alpha}$ is the outward unit vector normal to the spherical surface element:

$$
d S=r^{2} \sin \theta \mathrm{d} \theta \mathrm{d} \phi
$$

Evaluating this integral over the full coordinate range, we find the total energy of Martínez and Troncoso's solution within a sphere of radius $r$ to be:

$$
E=P_{0}=\frac{q^{4}}{2 r^{3}}+\frac{q^{2}}{r}+\frac{r}{2}-\frac{\left(q^{2}+r^{2}\right)^{3}}{2 l^{2} r^{3}}
$$

\section{Determining the energy by use of the Landau-Lifshitz pseudotensor}

We now turn to a second procedure for determining the local energy, the method of Landau and Lifshitz. One critique of Einstein's prescription is that it is not symmetric in its indices, thus precluding the definition of an angular momentum conservation law. Landau and Lifshitz strived to ameliorate this situation by finding a local measure of energy and momentum that is symmetric in its indices. The measure they found, although a pseudotensor, has the useful property of vanishing locally in inertial frames when expressed in quasiCartesian coordinates. When added to the stress-energy tensor it acts as a conserved current. Hence conservation laws for energy-momentum and angular momentum are each well-defined.

Based on these constraints, Landau and Lifshitz determined the energymomentum pseudotensor to be:

$$
L^{i j}=\frac{1}{16 \pi} S_{, k l}^{i j k l}
$$

where the $L^{i 0}$ represent the energy and momentum densities and:

$$
S^{i j k l}=-g\left[g^{i j} g^{k l}-g^{i k} g^{j l}\right]
$$


In similar manner to we can use Gauss's theorem to express the total energy as a surface integral:

$$
E=\frac{1}{16 \pi} \iint h^{00 \alpha} \mu_{\alpha} d S
$$

where the superpotentials $h^{00 \alpha}$ are defined as:

$$
h^{00 \alpha}=S_{, k}^{00 k \alpha}
$$

Substituting the metric components (12) into (27) and using (29) we find the relevant values of the superpotentials to be:

$$
\begin{aligned}
& h^{001}=\frac{q^{4}+2 q^{2} r^{2}+2 r^{4}}{r^{6}}-\frac{2 x\left(q^{2}+r^{2}\right)^{3}}{l^{2} r^{6}} \\
& h^{002}=\frac{q^{4}+2 q^{2} r^{2}+2 r^{4}}{r^{6}}-\frac{2 y\left(q^{2}+r^{2}\right)^{3}}{l^{2} r^{6}} \\
& h^{003}=\frac{q^{4}+2 q^{2} r^{2}+2 r^{4}}{r^{6}}-\frac{2 z\left(q^{2}+r^{2}\right)^{3}}{l^{2} r^{6}}
\end{aligned}
$$

We integrate this expression over the full range of coordinates for a sphere of radius $r$ and determine the total energy using the Landau-Lifshitz complex to be:

$$
E=\frac{q^{4}}{2 r^{3}}+\frac{q^{2}}{r}+\frac{r}{2}-\frac{\left(q^{2}+r^{2}\right)^{3}}{2 l^{2} r^{3}}
$$

This is identical to the expression obtained using Einstein's prescription.

\section{Using Papapetrou's energy-momentum complex}

Another energy-momentum complex, developed by Papapetrou, shares with the Landau-Lifshitz complex the advantage of being symmetric in its indices and thereby allowing the conservation of angular momentum to be well defined.

Papapetrou defined his energy-momentum complex to be:

$$
\Omega^{i k}=\frac{1}{16 \pi} N_{, a b}^{i k a b}
$$

where the functions $N^{i k a b}$ are given by:

$$
N^{i k a b}=\sqrt{-g}\left[g^{i k} \eta^{a b}-g^{i a} \eta^{k b}+g^{a b} \eta^{i k}-g^{k b} \eta^{i a}\right]
$$


and the $\eta^{a b}$ terms represent the components of a Minkowski metric of signature -2 .

Once more we use Gauss's theorem to express the total energy as a surface integral:

$$
E=\frac{1}{16 \pi} \iint \chi^{00 \alpha} \mu_{\alpha} d S
$$

with the superpotentials $\chi^{00 \alpha}$ defined as:

$$
\chi^{00 \alpha}=N_{, k}^{00 k \alpha}
$$

We determine the relevant values of the superpotentials to be:

$$
\begin{aligned}
& \chi^{001}=\frac{q^{4}+2 q^{2} r^{2}+2 r^{4}}{r^{6}}-\frac{2 x\left(q^{2}+r^{2}\right)^{3}}{l^{2} r^{6}} \\
& \chi^{002}=\frac{q^{4}+2 q^{2} r^{2}+2 r^{4}}{r^{6}}-\frac{2 y\left(q^{2}+r^{2}\right)^{3}}{l^{2} r^{6}} \\
& \chi^{003}=\frac{q^{4}+2 q^{2} r^{2}+2 r^{4}}{r^{6}}-\frac{2 z\left(q^{2}+r^{2}\right)^{3}}{l^{2} r^{6}}
\end{aligned}
$$

Performing the integral in (36) we once again obtain the same energy distribution found using the other two prescriptions, namely:

$$
E=\frac{q^{4}}{2 r^{3}}+\frac{q^{2}}{r}+\frac{r}{2}-\frac{\left(q^{2}+r^{2}\right)^{3}}{2 l^{2} r^{3}}
$$

\section{Conclusion}

We have determined that Martínez and Troncoso's solution representing a static, electrically-charged black hole with a minimally-coupled self-interacting scalar field belongs to the Kerr-Schild class. In applying the Einstein, Landau-Lifshitz and Papapetrou energy-momentum complexes to the metric, we have found that each yields an identical local energy distribution that depends on the electrical charge and cosmological constant. Qualitatively this differs from the standard Reissner-Nordström solution by the absence of a mass term and the negative energy value for large radii.

\section{Acknowledgements}

Thanks to K. S. Virbhadra for suggesting this problem and for his helpful advice. 


\section{References}

[1] S. S. Xulu, "The Energy-Momentum Problem in General Relativity," PhD Dissertation, University of Zululand, South Africa, 2002.

[2] A. Einstein, Preuss. Akad. Wiss. Berlin 47, 778 (1915).

[3] L. D. Landau and E. M. Lifshitz, The Classical Theory of Fields (Pergamon Press, Oxford, 1962), p. 341.

[4] A. Papapetrou, Proc. R. Ir. Acad. A 52, 11 (1948).

[5] S. Weinberg, Gravitation and Cosmology: Principles and Applications of the General Theory of Relativity (Wiley, New York, 1972), p. 165.

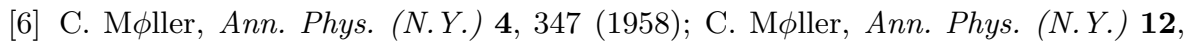
118 (1961).

[7] H. Bondi, Proc. R. Soc. London A427, 249 (1990).

[8] K. S. Virbhadra, Phys. Rev. D41, 1086 (1990); K.S. Virbhadra, Phys. Rev. D42, 1066 (1990); K. S. Virbhadra, Phys. Rev. D42, 2919 (1990).

[9] J. M. Aguirregabiria, A. Chamorro and K. S. Virbhardra, Gen. Rel. Grav. 28, 1393 (1996).

[10] N. Rosen, Gen. Rel. Grav. 26, 319 (1994).

[11] V.B. Johri, D. Kalligas, G.P. Singh and C.W.F. Everitt, Gen. Rel. Grav. 27, 323 (1995).

[12] N. Banarjee and S. Sen, Pramana J. Phys. 49, 609 (1997).

[13] I. Radinschi, Fizika B 9, 203 (2000).

[14] I. Radinschi, Chin. Jour. Phys.39, 231 (2001).

[15] S.S. Xulu, Int. J. Mod. Phys. A15, 4849 (2000).

[16] O. Aydogdu and M. Salti, Ap. \& S. S. 299, 227 (2005).

[17] M. Salti and A. Havare, Int. J. Mod. Phys. A20, 2169 (2005).

[18] P. Halpern, Ap. \& S. S.296, (2006).

[19] M. Salti, Ap. \& S. S. 299, 159 (2005).

[20] V. C. de Andrade, L. C. T. Guillen and J. G. Pereira, Phys. Rev. Lett. 84, 4533 (2000).

[21] M. Sharif and M. J. Amir, Mod. Phys. Lett. A22, 425 (2007). 
[22] E. C. Vagenas, Mod. Phys. Lett. A21, 1947 (2006).

[23] E. C. Vagenas, Int. J. Mod. Phys. A18, 5949 (2003).

[24] E. C. Vagenas, Int. J. Mod. Phys. D14, 573 (2005).

[25] E. C. Vagenas, Mod. Phys. Lett. A19, 213 (2004).

[26] S. S. Xulu, Int. J. Theor. Phys. 37, 1773 (2003).

[27] I. Radinschi and I. C. Yang, "On the Energy of String Black Holes," New Developments in String Theory Research, ed. Susan A. Grece, (New York: Nova Science, 2005)

[28] I. Radinschi and B. Ciobanu, "Weinberg Energy-Momentum Complex for a Stringy Black Hole Solution," (2006), gr-qc/0608029.

[29] R. M. Gad, Ap. \& S. S. 295, 459 (2005).

[30] A. Chamorro and K. S. Virbhardra, Int. J. Mod. Phys. D5, 251-256 (1994).

[31] E. C. Vagenas, Int. J. Mod. Phys. A18, 5781 (2003).

[32] S. S. Xulu, Int. J. Mod. Phys. D7, 773-777 (1998).

[33] P. Halpern, Mod. Phys. Lett. A21, 495 (2006).

[34] T. T. Bringley, Mod. Phys. Lett. A17, 157 (2002).

[35] R. M. Gad, Ap. \& S. S. 295, 451 (2005).

[36] K.S. Virbhadra, Phys. Rev. D60, 104041 (1999).

[37] S. S. Xulu, "Bergmann-Thomson energy-momentum complex for solutions more general than the Kerr-Schild class," (2007), gr-qc/0702066.

[38] R. Ruffini and J. Wheeler, Phys. Today 24, 30 (1971).

[39] J. D. Bekenstein, Phys. Rev. D5, 1239 (1972).

[40] C. Teitelboim, Phys. Rev. D5, 2941 (1972).

[41] T. Torii, K. Maeda and M. Narita, Phys. Rev. D64, 044007 (2001).

[42] E. Winstanley, Found. Phys. 33, 111 (2003).

[43] T. Hertog and K. Maeda, JHEP 0407, 051 (2004).

[44] T. Hertog and K. Maeda, Phys. Rev. D71, 024001 (2005).

[45] C. Martínez, R. Troncoso and J. Zanelli, Phys. Rev. D70, 084035 (2004).

[46] K. G. Zloshchastiev, Phys. Rev. Lett. 94, 121101 (2005). 
[47] G.Koutsoumbas, S. Musiri, E Papantonopoulos and G. Siopsis, JHEP 0610, 006 (2006).

[48] E. Radu and E. Winstanley, Phys. Rev. D72, 024017(2005).

[49] C. Martínez and R. Troncoso, Phys. Rev. D74, 064007 (2006).

[50] J. D. Brown, J. Creighton and R. B. Mann, Phys. Rev. D50, 6394-6403 (1994).

[51] J. P. S. Lemos, Phys. Lett. B352, 46 (1995).

[52] R. G. Cai and Y. Z. Zhang, Phys. Rev. D54, 4891 (1996).

[53] L. Vanzo, Phys. Rev. D56, 6475 (1997).

[54] D. R. Brill, J. Louko and P. Peldan, Phys. Rev. D56, 3600 (1997). 